\title{
Balanced Scorecard Et Incertitude Environnementale : Cas Des Entreprises Au Maroc
}

\author{
Mustapha Achibane \\ Azzouz Elhamma
}

Enseignant-chercheur, Ecole Nationale de Commerce et de Gestion (ENCG), Université Ibn Tofail - Kénitra

doi: 10.19044/esj.2016.v12n7p459 URL:http://dx.doi.org/10.19044/esj.2016.v12n7p459

\begin{abstract}
During the two last decades, the performance measurement systems are studied by a several researches in the developed countries. This type of researches is still absent in several developing countries such as Morocco for example.

From the results of an empirical research, this article examines the impact of the environmental uncertainty on the performance measurement systems of the 62 Moroccan firms. The principal results of this research show that the environmental complexity and uncertainty is identified as factor which tends to foster a more balanced approach.
\end{abstract}

Keywords: “Tableaux de bord” - Balanced scorecard - Environmental uncertainty.

\section{Résumé}

Durant les deux dernières décades, les systèmes de mesure de la performance ont fait l'objet de plusieurs travaux de recherche, notamment dans les pays développés. Or, ce type de recherches reste encore absent dans plusieurs pays en développement comme le Maroc par exemple.

À partir des résultats d'une recherche empirique, cet article examine l'impact de l'incertitude environnemental sur les systèmes de mesure de la performance de 62 entreprises installées au Maroc. Les résultats de cette recherche montrent que ces systèmes tendent à devenir plus « équilibrés » et se rapprochent du balanced scorecard lorsque l'environnement est incertain et dynamique.

Mots clés : Tableaux de bord - Balanced scorecard - Incertitude environnementale. 


\section{Introduction}

Actuellement, on assiste à ce qui est souvent appelé «crise » de contrôle de gestion (Bouquin, 1996). Cette crise touche notamment ses outils classiques dont les tableaux de bord centrés exclusivement sur le suivi des résultats financiers. Pour cette raison, de nombreux spécialistes ont proposé des modèles qualitatifs qui se sont traduits par l'intégration d'informations non financières (Chiapello et Delmond, 1994 ; Boisvert, 1991). Parmi ces nouveaux modèles, nous citons à titre d'exemple le balanced scorecard $(B S C)^{17}$, le modèle de Morin, Savoie et Beaudin (1994, 2001) et celui du « Skandia Navigator » (Edvinsson et Malone, 1999). Ces modèles intègrent en plus des indicateurs financiers, des indicateurs non-financiers donnant une image claire de la valeur « réelle » d'une société.

Or, ces tableaux de bord considérés comme « équilibrés » ne sont pas adoptés par toutes les entreprises. Cette situation s'est expliquée, par un courant de recherche, fondé sur la théorie de la contingence, par un certain nombre de facteurs explicatifs comme la taille (Elhamma, 2011 ; Germain, 2004 ; Hoque et James, 2000 ; etc.), la stratégie (Abernethy et Lillis, 1995 ; Perera et al., 1997; Gosselin et Dubé, 2002 ; etc.), la structure organisationnelle (Elhamma, 2011 ; Germain, 2004 ; etc.). Ce courant fourni actuellement un paradigme dominant de l'étude des systèmes de contrôle (Covaleski et al., 1996). Toutefois, les études portant sur l'impact de l'incertitude environnementale sur ces systèmes de mesure de la performance restent encore insuffisantes.

Dans ce contexte, le présent article tente de répondre à la question suivante : l'incertitude environnementale a-t-elle un impact significatif sur le caractère plus ou moins équilibrés des systèmes de mesure de la performance des entreprises au Maroc? Nous allons essayer de répondre à cette question dans le cadre de la théorie de la contingence.

Une définition des systèmes de mesure de la performance et un essai d'explication de l'impact de l'incertitude environnementale sur leur diversité (§1) et une présentation de nos choix méthodologiques (§2) sont nécessaires pour mener l'analyse statistique et de présenter les principaux résultats obtenus (§3).

\section{Contingence des systèmes de mesure de la performance et formulation de l'hypothèse}

Avant d'analyser l'impact de l'incertitude environnementale sur la diversité des systèmes de mesure de la performance (1.2), il nous paraît

17 Quelques traductions disponibles : «indicateur de performance global » (Kaplan et Norton, 1992), « tableau de bord équilibré » (Gervais, 2000). 
essentiel de définir notre champ d'analyse qui touche notamment le concept des systèmes de mesure de la performance (1.1).

\subsection{Systèmes de mesure de la performance: des tableaux de bord classiques au Balanced scorecard}

Les systèmes de mesure de la performance ont pour but principal de: " traduire la mission et la stratégie de l'entreprise en un ensemble d'indicateurs de performance qui constituent la base d'un système de pilotage de la stratégie " (Kaplan et Norton, 1996). Ils se présentent aujourd'hui sous plusieurs formes et appellations, mais les tableaux de bord et le balanced scorecard sont certainement les versions les plus répandues.

Selon Bouquin (2001, pp. 397-398), le tableau de bord se définit comme un instrument d'action composé d'un « ensemble d'indicateurs peu nombreux (cinq à dix) [sont intégrés] pour permettre aux gestionnaires de prendre connaissance de l'état et de l'évolution des systèmes qu'ils pilotent et d'identifier les tendances qui les influenceront sur un horizon cohérent avec la nature de leurs fonctions ». Plusieurs chercheurs insistent sur l'importance des tableaux de bord dits « équilibrés » ou « différenciés ». Des indicateurs de qualité, des mesures sociales, des indicateurs orientés clients et des indicateurs orientés processus doivent être également retenus pour compléter les mesures financières. Ces indicateurs non financiers permettent d'assurer la réactivité organisationnelle (Chiapello et Delmond, 1994), contribuent à la transversalité (De Montgolfier, 1994), et permettent de mesurer avec plus de pertinence que les données financières, la complexité organisationnelle (Mavrinac et Siesfeld, 1998).

Pour ces raisons, plusieurs types de tableaux de bord ont vu le jour. Des auteurs comme Edvinsson et Malone (1999) ont proposé le modèle du « Skandia Navigator » qui s’intéresse à mesurer le capital humain pour fournir des informations à destination de toutes les parties prenantes. Egalement, le modèle de l'European Foundation for Quality Management (EFQM) ou le modèle de la performance du Baldrige Quality Award (BQA). Selon Bollecker et Mathieu (2005), ces deux tableaux de bord « représentent les organisations comme des réseaux complexes de ressources, de stratégies, de productions, reliés par des causalités et des interactions mesurables (Oger et Pratt, 2002) », etc. Cependant, le BSC est certainement le système de mesure de la performance le plus connu sur le plan international (Bourguignon et al. 2002). Il a été développé par Kaplan et Norton (1992) aux Etats-Unis au début des années 1990. Il intègre en plus des indicateurs financiers, des indicateurs non-financiers qui sont regroupés autour de quatre axes : l'axe financier qui mesure le niveau et l'évolution des performances financières de l'entreprise ; l'axe « client » qui s’intéresse à regrouper les indicateurs qui permettent d'évaluer ce qui génère une satisfaction du client; l'axe 
« processus internes » qui concerne essentiellement la gestion des opérations et des processus peuvent contribuer à fournir un avantage concurrentiel à l'entreprise et l'axe «innovation \& apprentissage organisationnel » qui s’interroger sur la façon dont l'entreprise gère ses moyens humains et ses savoirs en vue d’atteindre ses objectifs stratégiques définis précédemment.

\subsection{Impact de l'incertitude environnementale sur les systèmes de mesure de la performance}

Plusieurs auteurs dont Fisher (1998) et Hartmann (2000) mettent en évidence que l'environnement constitue un facteur déterminant en ce qui concerne les caractéristiques des systèmes de contrôle de gestion en général et les systèmes de mesure de la performance en particulier. Gordon et Miller (1976) ont démontré que les entreprises évoluant dans un environnement incertain doivent augmenter la fréquence de parution de leurs rapports de gestion et intégrer des données diversifiées (non financières) pour faire face à ce type d'environnement. Dans le même sens, Gordon et Narayan (1984), Chenhall et Morris (1986), Chia (1995) mettent en évidence que les entreprises évoluant dans un environnement incertain font de plus en plus appel à des informations externes et non financières. Egalement, Chong and Chong (1997) expliquent que, pour faire face à l'incertitude environnementale, les entreprises ont besoin d'informations non financières.

De leur côté, Gosselin et Dubé (2002) démontrent que les entreprises poursuivant un comportement stratégique de type «prospecteur», qui évoluent dans un environnement caractérisé par un niveau élevé de l'incertitude, adoptent de plus en plus des indicateurs de mesure de performance non financiers par rapport aux autres entreprises poursuivant un comportement stratégique de type «défenseur » qui évoluent dans un environnement plus stable et moins complexe. Bescos et al. (2004) observent que les managers sont plus critiques vis-à-vis des budgets qui sont centrés exclusivement sur l'aspect financier de la performance lorsque l'incertitude perçue de l'environnement est élevée. Egalement, Germain (2004) constate que ce sont les entreprises qui perçoivent leur environnement comme étant dynamique et incertain qui détiennent la plus grande variété d'indicateurs de performance. Ceci conduit à poser l'hypothèse suivante :

Hypothèse de la recherche : Plus l'environnement est incertain et dynamique (ou certain et stable), plus les tableaux de bord sont «équilibrés » (ou «non équilibrés »).

\section{Méthodologie de la recherche}

Le cadre méthodologique nous permettra de présenter les caractéristiques de l'échantillon (2.1) et les variables de l'étude (2.2). 


\subsection{Echantillon de l'étude}

Afin de mener notre étude, un questionnaire a été construit et adressé à des entreprises installées au Maroc. Après une relance téléphonique et contacts physiques, soixante-seize (76) questionnaires ont été reçus, pour un taux de réponse brut de $18 \%$. De ce nombre, 14 questionnaires ont été éliminés pour diverses raisons, notamment les questionnaires incomplets, illisibles ou des entreprises non adoptant une comptabilité de gestion, etc. Le taux de réponse final a été de 15\%. Les questionnaires ont été remplis par 18 directeurs financiers (29\%), 23 contrôleurs de gestion (37\%), 17 comptables (27\%) et 4 autres types de responsables (6\%).

Deux principaux paramètres ont été utilisés pour décrire les caractéristiques des entreprises ayant constitué notre échantillon d'étude : secteur d'activité et effectifs des employés. Le tableau 1 ci-dessous présente le résumé des données sur ces deux paramètres.

Tableau 1: Caractéristiques de l'échantillon

\begin{tabular}{|l|c|c|c|c|c|}
\hline \multicolumn{3}{|c|}{ Secteur d'activité } & \multicolumn{3}{c|}{ Effectif du personnel } \\
\hline & Nombre & $\%$ & & Nombre & $\%$ \\
\hline Industrie & 48 & 77,42 & Moins de 200 & 30 & 48,39 \\
\hline BTP & 06 & 9,68 & Plus de 200 & 32 & 51,61 \\
\hline Services & 06 & 9,68 & - & - & - \\
\hline Commerce & 02 & 3,22 & - & - & - \\
\hline \multicolumn{1}{|c|}{ Total } & 62 & 100 & Total & 62 & 100 \\
\hline
\end{tabular}

Notre échantillon se compose de 48 entreprises industrielles (77\%), 6 entreprises du BTP (10\%), 6 entreprises des services (10\%) et 2 entreprises commerciales (3\%). Concernant la taille, 48\% de cet échantillon est constitué des PME et $52 \%$ des entreprises de grande taille.

\subsection{Variables de l'étude}

\subsubsection{La variable dépendante (équilibrage des tableaux de bord: EQTB)}

Pour opérationnaliser la variable relative à l'équilibrage des tableaux de bord, nous avons choisi les indicateurs proposés par Kaplan et Norton (1992):

- les résultats financiers (FNCE) ;

- la satisfaction des clients (CLNT) ;

- les processus internes (PRIN);

- et l'apprentissage organisationnel \& innovation (APOI).

Dans le questionnaire, il est demandé aux répondants d'indiquer, à l'aide d'une échelle sémantique différentielle à cinq points allant de «1 : degré d'intégration très faible » à " 5 : degré d'intégration très élevé », dans quelle mesure les principaux tableaux de bord de l'entreprise intègrent les indicateurs des quatre catégories mentionnées ci-dessus (plusieurs exemples 
d’indicateurs sont proposés). Plus le score global (sur 20 points) est élevé, plus le tableau de bord est « équilibré » et vice versa ${ }^{18}$.

\subsubsection{La variable indépendante (incertitude environnementale)}

Pour évaluer l'incertitude environnementale dans laquelle évoluent les entreprises étudiées, il a été demandé aux répondants d’exprimer leur degré d'accord sur différents items mesurés sur une échelle à supports sémantiques à cinq points. Ces cinq items proviennent de l'instrument de mesure élaboré par Gordon et Narayan (1984) et qui sont :

- «dynamisme de l'environnement économique » ;

- « dynamisme de l'environnement technologique » ;

- « prévisibilité de l’activité des concurrents sur le marché » ;

- « prévisibilité des goûts et des préférences des clients »;

- et « révision des politiques marketings ».

Pour les deux premiers items, le répondant avait le choix entre 5 réponses (allant de « 1 : très stable » à « 5 : très dynamique »). Pour les items $\mathrm{n}^{\circ} 3$ et $\mathrm{n}^{\circ} 4$, il y avait aussi 5 réponses (allant de « 1 : facilement prévisible » à « 5 : totalement imprévisible »). Enfin pour le dernier item, le répondant avait également 5 choix de réponses (allant de « 1 : très rarement » à « 5 : très souvent »). Plus le score global est élevé, plus l'environnement est incertain et dynamique et vice versa.

\section{Résultats et discussion}

Dans cette troisième section, nous allons présenter tout d'abord les premiers résultats de notre enquête relatifs à la nature des indicateurs intégrés dans les systèmes de mesure de la performance (3.1). Les résultats obtenus seront, ensuite, expliqués par l’incertitude environnementale (3.2).

\subsection{Nature des indicateurs intégrés dans les systèmes de mesure de la performance}

Le tableau 2 ci-après résume les résultats relatifs aux indicateurs intégrés dans les tableaux de bord utilisés par les entreprises étudiées.

Tableau 2 : Nature des indicateurs intégrés dans les systèmes de mesure de la performance des entreprises étudiées

\begin{tabular}{|l|c|c|c|c|}
\hline & Finance & Clients & $\begin{array}{c}\text { Processus } \\
\text { internes }\end{array}$ & $\begin{array}{c}\text { Innovation et } \\
\text { apprentissage }\end{array}$ \\
\hline Intégration très forte & $29 \%$ & $05 \%$ & $00 \%$ & $00 \%$ \\
\hline Intégration forte & $35 \%$ & $16 \%$ & $03 \%$ & $03 \%$ \\
\hline Intégration moyenne & $21 \%$ & $40 \%$ & $40 \%$ & $23 \%$ \\
\hline Intégration faible & $15 \%$ & $39 \%$ & $44 \%$ & $37 \%$ \\
\hline Intégration très faible & $00 \%$ & $00 \%$ & $13 \%$ & $37 \%$ \\
\hline Total & $\mathbf{1 0 0} \%$ & $\mathbf{1 0 0} \%$ & $\mathbf{1 0 0} \%$ & $\mathbf{1 0 0 \%}$ \\
\hline
\end{tabular}

${ }^{18}$ Cette démarche est largement inspirée de Germain (2004). 
La majorité des entreprises étudiées adoptent des tableaux de bord intégrant des indicateurs financiers (chiffre d'affaires, résultats, cash-flows, etc.). Plus de 64\% des entreprises intègrent «fortement » ou « très fortement » ces indicateurs dans leurs tableaux de bord. La proportion des entreprises qui adoptent des indicateurs relatifs aux clients est faible. Plus de $79 \%$ des entreprises ont déclaré qu'elles n’intègrent que moyennement ou faiblement ces indicateurs dans leurs tableaux de bord. Les indicateurs mesurant la performance relative aux processus internes sont faiblement intégrés dans les tableaux de bord des entreprises étudiées. En effet, 57\% des entreprises n'intègrent que faiblement ou très faiblement cette catégorie d'indicateurs dans leurs systèmes de mesure de la performance. Les indicateurs relatifs à l'innovation et à l'apprentissage sont presque absents dans les tableaux de bord des entreprises étudiées. 74,1\% des entreprises ont déclaré que cette catégorie d'indicateurs n’est intégrée que faiblement ou très faiblement dans leurs systèmes de mesure de la performance.

D’une manière générale, le contenu des tableaux de bord diffère d'une entreprise à une autre. Cette diversité pourrait être expliquée par un certain nombre de facteurs de contingence dont l'incertitude environnementale.

\subsection{Impact de l'incertitude environnementale sur l'équilibrage des tableaux de bord}

Pour identifier les types d'environnements dans lesquels opèrent les entreprises étudiées, nous avons utilisé l'analyse classificatoire. Elle permet de regrouper les pratiques similaires. Elle nécessite de choisir un algorithme de classification, c'est-à-dire de savoir la procédure adéquate pour regrouper convenablement des objets distincts dans des classes. Dans cette recherche, notre partition a été réalisée par classification hiérarchique. Le critère d’agrégation l'algorithme de Ward dont le but est de minimiser la variance interne de chaque classe et maximiser la variance entre classes a été utilisé. Par le biais de cette analyse classificatoire, nous avons identifié trois groupes d'environnements (tableau 3).

Tableau 3: types d'environnements dans lesquels opèrent les entreprises enquêtées

\begin{tabular}{|l|c|}
\hline \multicolumn{1}{|c|}{ Environnements } & $\mathbf{\%}$ \\
\hline Environnement très incertain et dynamique & 40,3 \\
\hline Environnement moyennement incertain et dynamique & 11,3 \\
\hline Environnement certain et stable & 48,4 \\
\hline Totale & $\mathbf{1 0 0}$ \\
\hline
\end{tabular}

Notre échantillon de l'étude se compose de 40,3\% des entreprises qui opèrent dans un environnement qualifié comme "très incertain et dynamique », 11,3\% de celles qui interviennent dans un environnement jugé comme "moyennement incertain et dynamique » et $48,4 \%$ des entreprises 
qui opèrent dans un environnement considéré comme « certain et stable ». Le tableau ci-après résume les résultats des moyennes relatives à la variable EQTB pour les entreprises en fonction de leurs environnements.

Tableau 4: Moyennes relatives à l'EQTB en fonction de l'incertitude environnementale

\begin{tabular}{|c|c|c|c|}
\hline & $\begin{array}{c}\text { Environnement } \\
\text { très incertain et } \\
\text { dynamique }\end{array}$ & $\begin{array}{c}\text { Environnement } \\
\text { moyennement incertain et } \\
\text { dynamique }\end{array}$ & $\begin{array}{c}\text { Environnement } \\
\text { certain et stable }\end{array}$ \\
\hline \multirow{2}{*}{$\begin{array}{c}\text { Moyennes } \\
\text { relatives à l'EQTB }\end{array}$} & 11,68 & 12,29 & 9,97 \\
\cline { 2 - 4 } & \multicolumn{3}{|c|}{ Toutes les entreprises } \\
\cline { 2 - 4 } & \multicolumn{3}{|c}{10,92} \\
\hline
\end{tabular}

Graphe 1 : Moyennes relatives à l'EQTB en fonction de l'incertitude environnementale

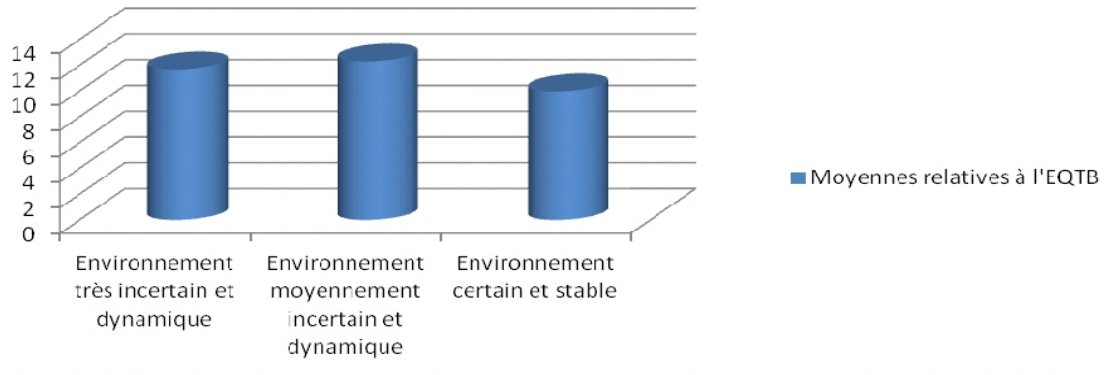

L’équilibrage moyen des tableaux de bord utilisés par les entreprises qui opèrent dans un « environnement très incertain et dynamique » et celles qui interviennent dans un «environnement incertain et dynamique » (respectivement 11,68 et 12,29) et plus élevé que celui observé dans les entreprises qui opèrent dans un « environnement certain et stable » $(9,97)$. Ces résultats soutiennent la validation de l’hypothèse de la recherche.

Pour tester l’hypothèse formulée ci-dessus, une régression linéaire simple sera utilisée. Nous l'avons choisie pour sa simplicité et sa large utilisation par les chercheurs en sciences de gestion. Le tableau 5 ci-dessous résume les résultats de ce test.

Tableau 5 : Résultats de la régression linéaire de l'équilibrage des tableaux de bord en fonction de l'incertitude environnementale

\begin{tabular}{|c|c|c|c|c|c|}
\hline $\begin{array}{c}\text { Variable indépendante } \\
\text { (incertitude } \\
\text { environnementale) }\end{array}$ & \multicolumn{5}{|c|}{ Variables dépendantes } \\
\cline { 2 - 6 } & FNCE & CLNT & PRIN & APOI & EQTB \\
\hline$\beta$ & 0.288 & 0,191 & 0,217 & 0,208 & 0,285 \\
t-statistic $^{2}$ & $2,325^{*}$ & $1,509^{\text {ns }}$ & $1,723^{* *}$ & $1,646^{\text {ns }}$ & $2,305^{*}$ \\
\hline $\mathrm{R}^{2}$ & 0.083 & 0,037 & 0,047 & 0,043 & 0,081 \\
\hline F-value & $5,407^{*}$ & $2,277^{\text {ns }}$ & $2,967 * *$ & $2,710^{\text {ns }}$ & $5,312^{*}$ \\
\hline
\end{tabular}

* Significatif au seuil de 5\% ** Significatif au seuil de $10 \%{ }^{\text {ns }}$ Non significatif

L'incertitude environnementale a un impact significatif et positif aussi bien sur les indicateurs financiers $(\beta=0,288 ; \mathrm{p}<5 \%)$ que sur ceux relatifs aux processus internes $(\beta=0,217 ; \mathrm{p}<10 \%)$. Par contre, les deux autres 
types d'indicateurs (des clients et de l'apprentissage organisationnel \& innovation) sont indépendants de ce facteur de contingence.

Généralement, l'incertitude environnementale a un impact positif et significatif au seuil de $5 \%$ sur l'équilibrage des tableaux de bord $(\beta=0,285$; $\mathrm{p}<5 \%$ ). La première variable explique environ $8,1 \%$ de la variation connue par la seconde variable. Le modèle identifié est statistiquement significatif au seuil de $5 \%(F=5,312)$. L'hypothèse de la recherche est donc validée par nos analyses. Plus l'environnement est incertain et dynamique (ou certain et stable), plus les tableaux de bord sont équilibrés et se rapprochent du BSC (ou non équilibrés et s’éloignent du BSC). Ce résultat confirme ceux obtenus par Gordon et Narayan (1984), Germain (2004), etc. Les entreprises qui évoluent dans un environnement qualifié comme incertain et dynamique ressentent le besoin d'accroître leur réactivité pour mieux faire face aux changements qui peuvent survenir dans leur environnement.

\section{Conclusion}

Cette recherche, réalisée auprès d'un échantillon de 62 entreprises installées au Maroc, montre que, comme cela avait été envisagé au début de la recherche, au regard des travaux portant sur la contingence du contrôle de gestion, l'équilibrage des systèmes de mesure de la performance est corrélé positivement à l'incertitude environnementale. Les tableaux de bord dits « équilibrés » sont adoptés davantage par les entreprises qui évoluent dans un environnement incertain et dynamique.

Les résultats obtenus doivent être interprétés avec prudence au vu des limites de notre recherche. Deux grandes limites d’ordre méthodologiques doivent être soulignées : la taille modeste de l'échantillon et le recours à une approche perceptuelle pour collecter les données.

Nous avons démontré ci-dessus que l'équilibrage des tableaux de bord est influencé par l'incertitude environnementale. Toutefois, il convient de signaler que d'autres facteurs organisationnels (stratégie, structure, etc.) et d'ordre humain participent aussi à la différenciation des systèmes de mesure de la performance (style de décision, culture du dirigent, etc.). Il nous paraît donc important de compléter notre présente étude par des autres qui tenteront de répondre aux problématiques soulevées.

\section{References:}

Abernethy M. A. et Lillis A.M. (1995), The impact of manufacturing flexibility on management control system design, Accounting, Organizations and Society, Vol. 20, n 4, pp. 241-258.

Bescos P.L., Cauvin E., Langevin P. et C. Mendoza (2004), Critiques du budget : une approche contingente, Comptabilité, Contrôle, Audit, 10 : 1, juin, pp. 165-185. 
Boisvert, H. (1991). Le contrôle de gestion : vers une pratique renouvelée, Editions du Renouveau Pédagogique Inc, Ottawa, Canada.

Bollecker M. et Mathieu P. (2005), L'évolution des systèmes de mesure de la performance vers la dimension sociétale: une lecture conventionnaliste, Cahier de Recherche n01/05, Centre d’Analyse et Recherche en Gestion des Organisations, Université de Haute Alsace.

Bouquin H. (1996), Pourquoi le contrôle de gestion existe-t-il encore ?, Gestion, Vol.21, $\mathrm{n}^{\circ} 3$, septembre.

Bouquin H. (2001), Le contrôle de gestion, Presses Universitaires de France, Paris, $5^{\text {ème }}$ édition.

Bourguignon A., Malleret V. et Norreklital H. (2002), L’irréductible dimension culturelle des instruments de gestion : l'exemple du tableau de bord et du balanced scorecard, Comptabilité-Contrôle-Audit/ Numéro spécial, mai, pp. 7-60.

Chenhall, R.H. et D. Morris (1986), The Impact of Structure, Environment, and Interdependence on the Perceived Usefulness of Management Accounting Systems, The Accounting Review, 66(1), pp. 16-35.

Chia, Y.M. (1995), Decentralization, Management Accounting Systems (MAS) Information Characteristics and Their Interaction Effects on Managerial Performance: a Singapore Study, Journal of Business Finance and Accounting, 22(6), pp. 811-830.

Chiapello E. et Delmond M.H. (1994), Les tableaux de bord de gestion, outils d'introduction du changement, Revue française de gestion, janvierfévrier, pp. 49-58.

Chong, V.K. et K.M. Chong (1997), Strategic Choices, Environmental Uncertainty and SBU Performance: A Note on the Intervening Role of Management Accounting Systems, Accounting and Business Research, 27(4), pp. 268-276.

Covaleski M.A, Dirsmith M.W. et Samuel S. (1996), Managerial accounting research: the contributions of organizational and sociological theories, Journal of Management Accounting Research, Vol. 8, pp.1-35.

De Montgolfier C. (1994), Structure et interactions des données pour le contrôle de gestion, Thèse de Doctorat en Sciences de Gestion, Université de Montpellier II.

Edvinsson L. et M.S. Malone (1999). Le capital immatériel de l'entreprise, Maxima Laurent du Mesnil Editeur

Elhamma A. (2011), Impact de la taille sur le contenu des tableaux de bord dans les entreprises au Maroc : résultats d'une étude empirique, Revue Marocaine de Recherche en Management et Marketing, $\mathrm{n}^{\circ} 4-5$, pp.317-329.

Elhamma A. (2011), Y-a-t-il un lien entre structure organisationnelle et contenu des tableaux de bord? Cas des entreprises au Maroc, Revue Management \& Sciences Sociales, n¹0/11, janvier-décembre, pp.71-86. 
Fisher J.G. (1998), Contingency theory, management control systems and firm outcomes: past results and future directions, Behavioral Research in Accounting, Supplement, Vol.10.

Germain C. (2004), La contingence des systèmes de mesure de la performance : les résultats d'une recherche empirique sur le secteur des PME, Revue Finance Contrôle Stratégie, Vol.7, mars, pp.33-52.

Gervais M. (2000), Contrôle de Gestion, Economica, Paris.

Gordon L.A. et Miller D. (1976), A contingency framework for the design of accounting information systems, Accounting, Organizations and Society, vol. $1, \mathrm{n}^{\circ} 1$, pp.59-69.

Gordon L.A. et Narayan V.K. (1984), Management accounting systems, perceived environmental uncertainty and organization structure: an empirical investigation, Accounting, Organization and Society, vol. 9, $\mathrm{n}^{\circ}$ 1, pp.33-47.

Gosselin M. et Dubé T. (2002), Influence de la stratégie sur l'adoption des mesures de performance en vigueur dans le système de comptabilité de gestion, $23^{\text {ème }}$ congrès de l'AFC, Toulouse, 16 et 17 mai.

Hartmann F. (2000), The appropriateness of RAPM: towards the further development of theory, Accounting, Organizations and Society, 25, 4-5, 451482.

Hoque Z. et James W. (2000), Linking Balanced Scorecard Measures to Size and Market Factors: Impact on Organizational Performance, Journal of Management Accounting Research, Volume 12, pp. 1-17.

Kaplan R.S. et Norton D.P. (1992), The balanced scorecard, measures that drive performance, Harvard Business Review, juanuary-february, pp. 71-79.

Kaplan R.S. et Norton D.P. (1996), The balanced scorecard, The Harvard Business School Press.

Mavrinac S. et Siesfeld A.G. (1998), La délicate mesure de l’immatériel, L'Expansion Management Review, décembre, pp. 31-38.

Morin E., Savoie A. et Beaudin G. (1994), L'efficacité de l'organisation. Théories, Représentations et Mesures, Chicotimi: Gaëtan Morin, Montréal.

Oger, B. et D.E. Pratt (2002), Modèles de création de valeur en Europe et aux Etats-Unis : une comparaison des modèles de l'EFQM et du Baldrige Award Criteria », Revue Comptabilité, Contrôle et Audit, mai, pp. 85-98.

Perera S., Harrison G. et Pooleet M. (1997), Customer-focused manufacturing strategy and the use of operations-based non-financial performance measures: a research note, Accounting, Organizations and Society, Vol. 22, $\mathrm{n}^{\circ}$ 6, pp. 557-572.

Savoie A. et Morin E. (2001), Développement récents dans les représentations de l'efficacité organisationnelle, Revue Psychologica, Universidade de Coimbra, Portugal, n²7, pp. 7-29. 